\title{
Mechanisms and In Vivo Functions of Contact Inhibition of Locomotion
}

Brian Stramer ${ }^{1}$ and Roberto Mayor ${ }^{2}$

${ }^{1}$ King's College London, Randall Division of Cell and Molecular Biophysics, London SE1 1UL, UK

${ }^{2}$ University College London, Cell and Developmental Biology Department, London WC1E 6BT, UK Email: brian.m.stramer@kcl.ac.uk ; r.mayor@ucl.ac.uk

\section{Preface}

Contact inhibition of locomotion (CIL) is a process of cellular avoidance upon migratory collision. It was initially characterized more than half a century ago and was at one time a widely studied model system to understand how cells migrate and dynamically interact. While CIL fell from interest for a number of decades, the scientific community has recently rediscovered this process. As a result, we are beginning to understand the precise steps of $\mathrm{CIL}$ along with the molecular mechanisms underlying this complex behaviour. Furthermore, this process is no longer just in vitro phenomenology; we now know from a number of different in vivo models that $\mathrm{CIL}$ is essential during embryogenesis.

Key words: contact inhibition, cell motility, collective cell migration, embryogenesis, patterning

\section{Introduction}

The term "contact inhibition" was first coined by the pioneering cell biologist Michael Abercrombie in $1954^{1}$. The discovery that numerous cell types undergo a "directional prohibition of movement" upon migratory collision was groundbreaking, not because the process was realized to be critical for animal physiology, but more to do with its ability to provide a framework to investigate the general mechanisms behind cell motility. Abercrombie spent his career studying contact inhibition of locomotion (CIL) and in doing so elucidated a number of fundamental aspects of cell migration, which forms the basis with regards to how the community thinks about the motility process to this day ${ }^{2}$. 
As will become clear in this review, the process of CIL is multifaceted, requiring not only an understanding about how cells migrate, but also how they interact and dynamically modulate their polarity, which are issues at the heart of almost all cell biological phenomena. However, for a number of reasons it fell from interest for a number of decades after Abercrombie's death in $1979^{3}$. First of all, the initial work on CIL stretched the boundaries of their understanding of cell motility (for example, cellular actin was only for the first time directly visualized in $1978^{4}$ ). Second, the function of CIL - if any - in animal physiology was totally unknown. These problems in understanding CIL are now being overcome. The field of cell motility has matured and our knowledge of the molecular mechanisms of cell migration is far greater than Abercrombie's time. Furthermore, live imaging of cellular behaviours in vivo is now commonplace. We are therefore armed with a far better knowledgebase to elucidate the mechanisms of CIL and its functions in processes such as animal development.

In this review, we outline our current understanding of the CIL process, and describe the quantitative assays at one's disposal to measure this phenomenon. We will discuss the recent mechanisms elucidated to play a role in CIL behaviours and discuss how these mechanisms mediate distinct steps of the CIL process. Finally, we will highlight the recently discovered functions of CIL in animal development and discuss as yet unexplored roles for this process during a variety of physiological processes.

\section{Types of CIL Behaviour}

Precisely what does it mean for a cell to undergo CIL? Lets start with what it isn't. While the term "contact inhibition" initially referred to effects on cell locomotion, it was adopted in the 60 s by those studying contact inhibition of proliferation. This led to confusion that persists to this day despite warnings from Michael Stoker, who pioneered the proliferative variety. In fact, Stoker preferred the term "density dependent inhibition of growth" for his process as there was evidence that physical contact was not needed to inhibit proliferation $^{5-7}$, and that "contact inhibition be restricted to arrest of movement as defined by Abercrombie and Heaysman" 8 . As of yet, there is no clear mechanistic connection between CIL and contact inhibition of proliferation and they should not be thought of as interrelated processes as some have suggested ${ }^{9}$.

Definition of CIL. So, confusion sorted. Not quite. Even Abercrombie's definition of CIL changed over his career as he and colleagues struggled to define its essence. The 
variability in the definitions of CIL used in current publications suggests the community is revisiting these exact same problems. A discussion of the history is therefore worthwhile or else we risk repeating it. In Abercrombie's final paper he defined it as "the phenomenon of a cell ceasing to continue moving in the same direction after contact with another cell." 10 While a complete loss of CIL involves "continued movement such as would carry one cell over the surface of another." 11 Note that his definition is purposefully vague with regards to what happens after contact. Does the cell simply stop moving? Does it repolarize and migrate away? Is it randomly deflected from the colliding cell? Any of these behaviours constitute CIL by this simple definition. Other CIL researchers noted with obvious consternation that 'quite a number of phenomena having to do with cells' influences upon one another's movements have come to be regarded as expressions of contact inhibition. However, no single, central mechanism has been shown to underlie them all"12. Even a cessation of leading edge dynamics upon collision, which is often assumed to be a hallmark of CIL, was realized to be a poor predictor of contact inhibition ${ }^{12-15}$, with Abercrombie even stating that leading edge paralysis was a "red herring" with regards to the underlying mechanism of $\mathrm{CIL}^{14}$.

Variability in CIL responses. To clarify CIL behaviours efforts were made to classify it into specific types with some envisioning up to 6 different CIL responses ${ }^{12}$. In contrast, Abercrombie simplified CIL into two types that involve either: (I) the local paralysis and contraction of the leading edge (with contraction being the defining response ${ }^{15,16}$ ) or (II) the difficulty for a cell to move across the surface of another because it may simply be less adhesive than the substratum ${ }^{16,17}$. To take this a step further with regards to the final outcome of the response, which is critical to understand the physiological roles of CIL (see section on Embryological functions of CIL), Type I CIL would lead to an active movement away from the colliding partner (supplementary movies 1 and 2) whereas Type II CIL results in a simple cessation of movement or a random deflection (supplementary movie 3, Figure 1A, B). It is possible that Type II CIL is a more passive response and controlled by the mechanics of the collision as membrane tension and external forces are known to effect the actin polymerization machinery at the leading edge ${ }^{18-20}$. In contrast, Type I CIL must involve active repolarisation signals and is likely also controlled by specific surface receptors that allow for cell-cell recognition, which may not be required for Type II behaviour. Regardless, it is important to stress that CIL does not constitute a single behaviour making its quantification quite complex (Box 1). Even within a homogenous population of cells one may see a range of collision outcomes, which may be due to the 
variability of the response or the orientation of the colliding partners (e.g. lamellae to lamellae vs. lamellae to rear $)^{11,14,21-23}$. CIL is therefore not a binary behaviour; changing the geometry of the collision or the signals involved in the interaction may lead to a different type of CIL response entirely. A complete failure in CIL (i.e. cells crawling on top or beneath of one another) has only been observed in a few cell types, with most involving cancer cell interactions with normal cells ${ }^{11}$ (supplementary movie 4).

Another parameter that can modify CIL behaviour involves heterotypic vs. homotypic interactions (Figure 1C, D). There are many examples where collisions between cells of the same type (homotypic CIL) yield completely different outcomes compared with collisions with other cells (heterotypic CIL). On the one extreme, one of the cells in a heterotypic collision may be completely defective in CIL and use the other as a substrate for its motility. Alternatively, a heterotypic response may simply be subtly changed compared with the homotypic response, yielding a different type of CIL behaviour. Importantly, the ability of heterotypic collisions to yield non-mutual outcomes, gives the CIL process an added level of instructive power, which we will see can lead to emergent cellular behaviours during animal development (see below).

\section{Stages of the CIL response}

In order to elucidate the mechanisms controlling CIL it is important to understand the possible regulatory stages of the process. For the purpose of this review we will divide the response into four stages, which have some basis from experimentation. This is a somewhat arbitrary division as these steps overlap in time. Furthermore, it is important to note that not all cells undergoing CIL will experience all of these behaviours. For example, the final outcome of Abercrombie's Type I CIL is cell contraction and separation, which is very different from the non-repolarizing Type II response (as discussed above). Therefore different CIL responses are likely to feature distinct stages. We will focus on hypothetical steps of classical Type I CIL, which Abercrombie thought to be the prototypical CIL behavior $^{16}$. However, it is possible that other CIL types (e.g. Type II) simply represent an abbreviated response involving some of these stages (e.g. missing the last step of cells migrating away), and this framework is useful for anyone studying CIL regardless of the response type.

The proposed sequence of events implicated in CIL is outlined in Figure 2 and includes the following steps: 1) cell-cell contact, 2) inhibition of cell protrusive activities at the site of contact, 3 ) contraction of protrusions at the cell contact and generation of a new 
protrusion, and 4) migration away. The first step of cell-cell contact is essential for CIL and distinguishes it from other repulsive processes that involve cell responses at a distance (e.g. chemorepulsion). Here it is important to differentiate between cell contacts established during head-to-head collisions (lamellae to lamellae) versus other orientations as the geometry of the collision can affect the outcome ${ }^{11,14,21-23}$. This initial contact is followed by a variable degree of protrusion inhibition. In many cases it is possible to observe a cessation of protrusion activity immediately after contact ${ }^{24,25}$, however as mentioned earlier, this is not necessarily correlated with CIL capacity. Subsequently, the cell will undergo leading edge contraction and cell repolarization, although the precise order of these two events is possibly dependent of the type of CIL. While in some cell types contraction of lamellae occurs prior to cell repolarization ${ }^{1,26}$, others have observed the opposite ${ }^{27}$. The final step of a stereotypic CIL response is migration away from the collision, which likely involves a re-activation of the migratory machinery that may have been affected by the initial collision.

\section{Molecular mechanisms of CIL}

While there is diversity in CIL behaviours, we are starting to get a clearer picture of the basic molecular mechanisms underlying the various steps behind this phenomenon. Here we discuss recently elucidated mechanisms underlying the distinct stages of the response highlighted in Figure 2.

Establishing cell-cell contact. It is clear that cell-cell adhesion plays an important role in CIL, although the molecular nature of the adhesion is unclear in many cell types ${ }^{26-29}$. Recent evidence has revealed that the initial cell contact during CIL often involves the formation of a transient Cadherin-mediated intercellular junction. Cadherins are a family of transmembrane glycoproteins, normally thought to be associated with epithelial monolayers, which facilitate calcium-dependent cell-cell adhesion. Different Cadherins are found at the cell-cell contact during CIL in a range of cell types, such as E-cadherin, $\mathrm{N}$ cadherin and cadherin 1127,30-35. Furthermore, numerous proteins involved in the formation of a stable adherens junction are also found to be involved in CIL, such as $\beta$ catenin, $\mathrm{p} 120$, vinculin and $\alpha$-catenin ${ }^{27,32,36}$. It is not completely clear why these adherens junctions are transient during CIL, while having almost the same composition as a stable junction between epithelial cells. Recent work using Xenopus and zebrafish 
neural crest cells has shed some light on this problem by comparing junctional formation during responses that involve either a stable or transient adhesion. Cells specifically expressing $\mathrm{E}$-cadherin remain in contact, while cells expressing $\mathrm{N}$-cadherin undergo repulsion ${ }^{27}$. The main difference here is that in contrast to $\mathrm{E}$-cadherin, $\mathrm{N}$-cadherin signalling leads to cell repolarization ${ }^{27}$ (Figure 3), which is associated with the differential capacity of E-cadherin to bind to $p 120^{27}$. It remains to be seen if Cadherin switching is a general mechanism to control CIL capacity. However it is interesting to note that the switch between $\mathrm{E}$ - and $\mathrm{N}$-cadherin is observed during epithelial mesenchymal transition (EMT) in neural crest and cancer cells ${ }^{27,37}$, which may aid their invasiveness through modulation of CIL behaviours (see Unexplored roles for CIL). Furthermore, the differential binding of E- and N-cadherin to p120, which modulates CIL capacity in neural crest cells, is also linked to the invasive potential of pancreatic cancer cells ${ }^{38}$.

In addition to classical cell adhesion molecules, other families of receptors have been implicated in establishing the initial contact during CIL. Eph receptors are a group of tyrosine kinase receptors that bind transmembrane ephrin ligands on neighbouring cells, and bidirectional signalling from Eph-ephrin interactions can lead to a repulsive response $^{39}$. In Cajal-Retzius neurons both EphA and EphB receptors are required for normal CIL behaviour, which is necessary for their dispersion ${ }^{40}$. EphA is also involved in homotypic CIL between prostate cancer cells, while EphB acts as a suppressor of heterotypic CIL between these cells and normal fibroblasts ${ }^{41,42}$. Thus, the balance of signalling mediated by different Eph receptors can determine a cell's capacity for CIL. This is intriguing as the loss of CIL upon contact of cancer cells with normal cells is speculated to play a role in cancer metastasis ${ }^{10}$, and in prostate cancer cells it has been shown to occur through modulating Eph-ephrin signalling ${ }^{41,43}$. There may also be more complicated interactions between the various receptors involved in CIL. Crosstalk between Ephs and Cadherins controls ${ }^{44}$. Furthermore, similar crosstalk has been observed during embryonic boundary formation in Xenopus mesoderm ${ }^{45}$, which is hypothesized to involve a process analogous to CIL (see section on Unexplored roles for CIL).

Recently the receptor Robo and its ligand Slit have also been revealed to be involved in CIL between fibroblasts. Robo receptors belong to the immunoglobulin (Ig) superfamily of cell adhesion molecules (CAMs), and Slits function as their normally secreted ligands. NIH 3 T3 mouse fibroblasts utilize Robo4-Slit2 signalling during CIL, with Slit2 apparently tethered to the cell surface rather than secreted. Similar to Ephs, crosstalk between Slit-Robo and Cadherins is possible ${ }^{46,47}$, suggesting that receptor interactions may be a common theme during CIL. 
Protrusion inhibition. The CIL response is normally initiated by interactions between actin-rich lamellae of colliding cells ${ }^{16,25,26}$. One of the main regulators of lamellae dynamics during cell migration are small GTPases, Rho, Rac and Cdc42, which have also been implicated in CIL. For example, collision of neural crest cells leads to an inhibition of Rac1 activity ${ }^{25,48}$, which is dependent on $\mathrm{N}$-cadherin and Wnt-planar cell polarity (PCP) signaling ${ }^{25,49}$. Upon collision many PCP components, including Dishevelled, Prickle1 and Strabismus, are recruited to the receptor Frizzled7 at the cell-cell contact leading to the inhibition of Rac1 and activation of RhoA ${ }^{25,27,49}$ (Fig. 2 [Protrusion inhibition]). It is not clear precisely how Rac1 is inhibited, but it may occur indirectly through RhoA activation as these GTPases are known to be antagonistic ${ }^{50}$. In addition, in neural crest cells Par3 becomes localized to the cell contact, which is thought to inhibit the Rac1 activator Trio ${ }^{48}$ and this may also help to inhibit Rac1 (Fig. 2 [Protrusion inhibition]). Similarly, homotypic CIL between pancreatic cancer cells is controlled by activation of RhoA-ROCK via EphA activation, while loss of $\mathrm{CIL}$ during heterotypic prostate cancer cell/fibroblast interactions involves EphB3 or B4 activation and induction of Cdc42 leading to continued cell migration ${ }^{41}$. Finally, in fibroblasts Robo4-Slit2 induction during CIL controls the duration of Rac1 activity ${ }^{51}$ and glial cell CIL is dependent on Rac1 regulation by Tiam $1^{52}$. Thus small GTPase regulation is a conserved aspect of CIL regulation in numerous cell types.

Contraction of protrusions and repolarization. Acto-myosin mediated contraction of protrusions is often observed during CIL. Similar to inhibition of protrusion activity, small GTPases also play an essential role in this stage of the response. In fibroblast and Xenopus neural crest cells this occurs through RhoA-ROCK activity ${ }^{25,53}$ and in Drosophila macrophages involves the RhoA responsive formin, Diaphanous ${ }^{26}$. Abercrombie in his initial discovery of CIL hypothesized a buildup in intercellular lamellar tension ${ }^{54}$, which has recently been revealed to control CIL between Drosophila macrophages ${ }^{26}$. In Drosophila macrophages, it was revealed that the development of contractile stress involves the physical coupling of the flowing actin networks in colliding lamellae suggesting a mechanochemical signalling mechanism ${ }^{26}$. This tension could subsequently have a direct effect on cell repolarization. For example, in mesodermal cells generating tension by pulling with a $\mathrm{C}$-cadherin coated magnetic bead can induce cell repolarization ${ }^{55}$. As mesodermal cells are known to undergo $\mathrm{ClL}^{56,57}$, it would be interesting to determine if tension during their $\mathrm{CIL}$ response is actually required for their reorientation. 
In addition to actin, other cytoskeletal elements such as microtubules have also been implicated in CIL in numerous cell types ${ }^{26,48,53,58,59}$. In Drosophila macrophages microtubule targeting of the cell-cell contact is a hallmark of the response $\mathrm{e}^{26,58}$, and in fibroblasts and neural crest cells, engagement of the cell-cell adhesion during CIL induces microtubule catastrophe, which seems essential for cell repolarization ${ }^{48,53,59}$. However the precise mechanisms by which microtubules control the CIL process is currently unclear.

It should be noted that the precise sequence of the events of contraction and repolarization is not clear. While in chick heart fibroblasts and Drosophila macrophages, protrusion collapse appears to precede the formation of new protrusions away from the contact site ${ }^{15,26,28}$ (Fig. 2 [3b]), this may not always be the case. In neural crest cells, the formation of new protrusions precedes loss of the cell-cell junction and this repolarization is thought essential to help generate the tension needed for subsequent lamellar contraction and cell separation ${ }^{27}$ (Fig. 2 [3a]).

Migration away from the collision. Once the cells contract their protrusions and repolarize, the final step in a prototypical Type I CIL response is to migrate away. One therefore could speculate that there must be reorganization of cell-substrate traction stresses for this to occur involving a modification of integrin adhesions. Abercrombie was actually one of the first to visualize focal adhesions during cell motility using interference reflection microscopy, which highlights regions of the cell membrane in contact with the substratum ${ }^{22}$. However, using this technique in fibroblasts undergoing $\mathrm{CIL}$ he did not observe any gross change in adhesions prior to cell contraction ${ }^{22}$, despite some earlier evidence to the contrary ${ }^{60}$. On the other hand, Xenopus neural crest cells reduce focal adhesions in the vicinity of cell-cell contacts as measured by observing changes in the distribution of focal adhesion components, such as FAK and Paxillin ${ }^{27,61}$. This reduction of focal adhesions at the cell-cell contact site would then lead to a redistribution of the cellmatrix traction stresses necessary for movement away from the colliding partner (Fig. 2 $[3,4]$ ). These observations suggest that focal adhesion reorganization during CIL might be a cell-type dependent phenomenon or may occur in only some types of CIL. Alternatively, the failure to observe focal adhesion changes in fibroblasts by Abercrombie may have been the result of limitations in the technique used to observe adhesions in these cells. Nevertheless, it is likely that there is an interaction between cell-cell and cell-matrix adhesions during $\mathrm{CIL}$ as both must be regulated for cells to successfully separate and 
migrate away. Indeed, there are many examples of crosstalk between these adhesion complexes in a number of cell types ${ }^{62-68}$.

We have clearly come a long way since CIL's initial discovery in terms of understanding the regulation of its various stages as numerous components have been identified to be involved in the process (Fig 2 [Table 1]). What is clearly missing is how all of these diverse regulatory factors, from Cadherins to focal adhesions, are integrated to induce a seamless response, and whether all cell types (or CIL types) share these same mechanisms.

\section{Embryological Functions of CIL}

While CIL is at first appearance a simple interaction it has the power to play an instructive role when present within a population of cells. Indeed, CIL is an excellent example whereby simple rules can lead to emergent behaviours, which is a recurring theme in biological pattern generation. Indeed, numerous mathematical models have revealed the ability of CIL to generate patterns of cellular movements in vitro (Box 2). However, as we will see, CIL is no longer just an in vitro phenomenon, as a number of groups have recently revealed roles for this process during embryonic development.

Driving cellular dispersion. CIL has the capacity to disperse a cell population such that the cells are driven into free space (supplementary movie 5) in a process Abercrombie termed "negative taxis" 69 . There are many instances during embryogenesis where a population of cells originates in a specific location and subsequently disperses to reach their final positions, and CIL can play a role in their spreading. This is indeed what occurs during migration of Cajal-Retzius cells (Figure 4A). These cells are born in distinct regions of the brain and spread throughout the cerebral cortex, which is critical as they control the subsequent migration of other cell types. CIL dynamics between Cajal-Retzius cells, regulated by Eph-ephrin signalling, is sufficient to induce the spreading and final distribution of the population ${ }^{40}$. Repulsion between these cells is essential for their distribution, suggesting that they must be undergoing a Type I kind of CIL behaviour.

Inducing cellular tiling. While Cajal-Retzius cells distribute relatively evenly throughout the cerebral cortex, their distribution is not completely homogenous ${ }^{40}$. However, there are 
many instances in the embryo where cells adopt a very even distribution such as tiled arrays, and CIL can be a driving factor. Drosophila macrophages are an example of a cell type that disperses during embryogenesis, eventually adopting a more homogenous distribution akin to tiling ${ }^{58}$ (Figure 4B). A combination of live imaging (supplementary movie 2) and mathematical modelling showed that CIL is sufficient to explain this even spacing between Drosophila macrophages ${ }^{58}$. The difference between CIL inducing simple spreading of a population versus cell tiling appears to be related to the precision of the CIL response. CIL between Drosophila macrophages is highly orchestrated and collision dynamics are synchronized between colliding cells in terms of changes in cell motion. This precision is essential for their even spreading ${ }^{26}$. Mathematical modelling suggests that random deflections (e.g. as a result of a Type II CIL response) leads to reduced homogeneity of the population and inhibiting the orchestration of Drosophila macrophage CIL prevents cell patterning ${ }^{26}$. It has been noted that Cajal-Retzius cell distribution shows regions of aggregation ${ }^{70}$, which would be somewhat predicted by decreasing the CIL precision, and it is therefore possible that imprecise repulsive interactions are actually instructive for the final density of these aggregations.

Coordinating collective cell migration. As mentioned earlier, in vitro analysis of CIL suggests that it can help orchestrate the collective migration of a cell population, and neural crest migration during embryogenesis is an excellent example of this coordinating influence (Figure 4C). Different neural crest populations migrate as coherent clusters or in linear chains during development, and in vivo experiments in both Xenopus and zebrafish have revealed that in the absence of CIL coherent movement of cell clusters is severely affected $^{25}$. Furthermore, in vitro analysis of epithelial cell migration coupled with mathematical modelling revealed that chain migration also emerges solely through CIL dynamics $^{23}$. Cranial neural crest, for example, migrates toward a chemokine source (Sdf1), and the presence of CIL within the population is thought to restrain protrusion between neighbours, thus allowing the entire population to acquire a single, coherent polarity necessary for their collective motion ${ }^{49}$.

Interestingly, heterotypic CIL interactions between Xenopus and zebrafish neural crest and other cell types are also instructive during embryogenesis. For example, neural crest aid in the morphogenesis of epithelial placodes, which contribute to sensory organs (Figure 4D). The neural crest cells are attracted to the placodes by placodal expression of Sdf1. However, placodal cells undergo CIL in response to the neural crest cells, and the former are subsequently repelled inducing a "chase-and-run" behaviour. The neural crest 
cells also undergo CIL in response to placodal contact, however their repolarization appears to be overridden by their attraction to Sdf1 - thus allowing the chase to continue $^{61}$. This cooperation between $\mathrm{CIL}$ and chemotaxis has also been observed in breast cancer cells in vitro ${ }^{71}$, suggesting that CIL integration with chemotactic cues may be a common theme in directing cell movement.

It is interesting to speculate why CIL in some cells leads to collective motion while in others to cell dispersion. It is possible that the final outcome is related to specific behaviours associated with collision geometry; modelling has revealed that if CIL capacity is reduced during head-to-tail interactions collective motion can emerge within a cell population $^{21,23}$. However, this may be cell-type dependent as Drosophila hemocytes fail to undergo CIL during head-tail interactions and disperse rather than show collective migration $^{26}$.

\section{Unexplored Roles for CIL}

There are numerous physiological processes where cellular behaviours suggest roles for CIL. Notably, some of these are not actually that speculative as they involve stereotypical CIL responses that so far have not been linked to the phenomenon of CIL. Other 'speculative' roles were even historically called CIL (presumably during a time when studying CIL was in vogue), but have undergone recent nomenclature changes such that they are now considered distinct processes. Below are just a few examples.

Neuronal contact repulsion. The nervous system is a good example where CIL is likely playing a role during a number of developmental events. Neuronal pathfinding is known to involve both positive cues (such as chemotactic signals) as well as negative, repulsive signals ${ }^{72}$. Some of the repulsive varieties that neuronal growth cones experience during their guidance are known to involve direct cell-cell contact and this has for the last decade been termed 'contact repulsion'. However, during Abercrombie's time this same 'contact repulsion' process was realized to be CIL behaviour ${ }^{73-76}$.

More speculative roles for CIL in the nervous system involve neuronal patterning (Figure 5A). There are examples where neuronal populations develop into evenly spaced cellular arrays, such as in the retina ${ }^{77}$, that one could imagine involves a similar CIL process to the tiling of Drosophila macrophages. Indeed, cell-cell contact induced repulsive interactions have been shown to play a role in neuronal tiling ${ }^{78}$. Similarly, the development of dendritic fields, which involve spacing of not only neuronal cell bodies, but 
also their dendritic projections, also require contact-mediated mutual repulsion that one could consider to be a CIL response ${ }^{79,80}$.

Zebrafish stripe formation. The intricate patterning of zebrafish pigment cells involves a process of cell repulsion that for all intent and purposes appears to be the same phenomenon (Figure 5B). The stripes in zebrafish skin are composed of three cell types (melanophores, xanthophores, and iridophores) that tile into cellular arrays as well as segregate to form distinctly coloured lines. During stripe development, the pigment cells are migratory and undergo contact-dependent repulsive behaviour, which is essential for acquisition of the final pattern ${ }^{81-83}$. Furthermore, repulsive responses are unique depending on whether the pigment cell is interacting with a cell of its own type or another, suggesting that stripe formation is an example where homotypic versus heterotypic CIL responses are also playing a role ${ }^{81,84}$. Interestingly, this heterotypic pigment cell repulsive response involves xanthophores chasing melanophores in a process the authors termed "run-and-chase" behaviour ${ }^{81}$, which looks identical to the CIL-induced "chase-and-run" interaction between neural crest and placodal cells ${ }^{61}$.

Boundary formation. During embryogenesis, cells within tissues, such as in the hindbrain, are often segregated into distinct subdivisions in which cells are prevented from intermingling with neighbours at subdivision borders (Figure $5 \mathrm{C}$ ). The generation of these boundaries is controlled by a number of active processes, such as differential adhesion, the generation of cortical tension at the interface, and cell repulsion ${ }^{85}$. This repulsive response (actually termed CIL in recent papers ${ }^{45,85,86}$ ) is known to involve Eph-ephrin signaling ${ }^{45,87}$, which as discussed earlier, is a critical player in CIL in a number of other cell types.

Cell condensation. There may be instances where turning off CIL behaviours may be instructive for a population of cells. During embryogenesis groups of mesenchymal cells often need to condense in order to begin forming a coherent tissue, such as in cartilage development ${ }^{88}$ (Figure 5E). Assuming that the cartilage precursor cells due indeed spread via CIL interactions (as one may expect as at least some cartilage is neural crest derived), one aspect of the aggregation signal within the population may involve reducing the repulsive responses between the cells. 
Inflammatory cell recruitment. It is not just embryogenesis where CIL may be playing a role. The immune system involves a complex ecosystem of cell types that requires the dynamic regulation of cell interactions, and is also a good place to look for instructive roles for CIL. Indeed, one of the few migratory interactions that have been shown to completely fail in CIL is the collision between leukocytes and fibroblasts ${ }^{89,90}$, and it is possible that this failure in heterotypic CIL is essential to allow leukocyte migration to sites of infection or wounds (Figure 5D). Interestingly, leukocytes still show CIL responses during collisions with each other ${ }^{89}$ and the negative regulation of this homotypic CIL behaviour may also be playing a role during immune activation. As discussed above, Drosophila macrophages spread themselves out uniformly within the animal ${ }^{26,58,91}$, which may be essential for even immune system coverage. However, during wound activation they must aggregate at wound sites by somehow modifying their normal repulsive behaviour ${ }^{92}$, and recent modelling of Drosophila macrophage wound responses revealed that dampening CIL was essential for their recruitment ${ }^{93}$. It remains to be determined whether mammalian immune cells have the capacity to modulate their CIL responses, but it is interesting to speculate that processes such as immune cell swarming during infections ${ }^{94}$ involve changes in repulsive behaviour to allow such cell aggregation.

Cancer metastasis. Abercrombie speculated about roles for CIL in animal physiology

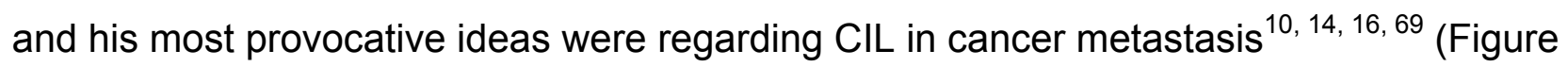
$5 \mathrm{~F})$. He discovered that many cancer cells lost their ability to undergo CIL - not to each other as many have incorrectly stated - but to other normal cells ${ }^{69}$. Indeed, he noted that total failure in contact inhibition has only been observed in a few heterotypic interactions, such as sarcoma cells with fibroblasts ${ }^{11}$ (supplementary movie 4 ). It must be clear that he did not suggest cancer cells lost their CIL capacity entirely as many cancer cells still maintain homotypic CIL. He therefore realized that homotypic repulsion between cancer cells "would greatly increase the efficiency with which the population spreads"69 (supplementary movie 5). It is interesting to hypothesise that metastatic processes such as EMT, which involve a loss of epithelial characteristics and an acquisition of mesenchymal traits (such as the possibility for an enhanced CIL capacity), could be playing a role in the initial spreading of the cancer, similar to neural crest cells ${ }^{27}$ (Figure 3). Despite much speculation about CIL in cancer, the molecular mechanisms involved in modulating CIL behaviour during homotypic and heterotypic cancer cell interactions is largely unexplored. For example, it is currently unclear whether the $\mathrm{E}$ - to $\mathrm{N}$-cadherin switch (Figure 3 ), which is widely observed during EMT in cancer, plays a role in modulating CIL behaviour during 
metastasis. Furthermore, despite a number of recent in vitro studies confirming a loss of heterotypic CIL in cancer cells ${ }^{41,42,52}$, it still remains to be seen whether this is also occurring in cancers in vivo. As cancer progression is now amenable to live imaging ${ }^{95}$, it is time to revisit Abercrombie's ideas.

\section{Conclusion}

It is clear that the process of CIL is being rediscovered, both in terms of a model to address fundamental cell biology, as well as a 'signal' to control the movement of cells during embryogenesis. While we have come a long way in terms of discovering molecular pathways controlling CIL, what is currently missing is an understanding of how all these factors are coordinated, and importantly, fit into the overall dynamics of the process. CIL behaviour, particularly the active Type I variety cannot be explained by simple signalling paradigms, and will involve more complex mechanochemical processes that modulate both cell motility and subsequent repolarisation. The challenge will be in understanding how rapid cytoskeletal and signalling dynamics propagates both spatially and temporally in the cell to lead to ultimate cell repolarization. This is not trivial as it involves bridging temporospatial scales which is experimentally and theoretically challenging, and something that the cell motility field in general is currently struggling with ${ }^{2}$. Furthermore, as CIL has recently been identified in a diverse range of cell types we are starting to revisit a problem that Abercrombie and colleagues came across decades ago, which is related to its precise definition. It may be that Abercrombie's final definition, 'a cessation of forward motion upon migratory collision', is adequate. However, as this definition encompasses such a wide variety of ultimate responses, it may become so broad that it is rendered useless on its own. One solution is to reconsider the idea that there are 'types' of CIL responses, which may each have distinct steps and regulatory mechanisms. What will help in this classification endeavour is precise characterisation of CIL behaviours in different contexts. Indeed, the more CIL behaviours that are identified the easier it will be to categorize the response and extrapolate mechanisms, and functions, from one cell type to the next. 


\section{Acknowledgments}

We thank Gareth Jones, Claudia Linker, and Maddy Parsons for comments on the manuscript. BS is supported by the Wellcome Trust and the European Research Council (ERC) under the European Union's Horizon 2020 research and innovation programme (grant agreement No. [68108]). RM is supported by the Medical Research Council (MRC) and the Biotechnology and Biological Sciences Research Council (BBSRC). 


\section{Box 1| CIL Assays (includes Figure of assays)}

The variability in CIL behaviours makes it essential to choose the correct assay, with multiple required to fully describe the phenomenon:

Mixing assay. Two differentially labelled explants are cultured at short distance and overlapping upon outgrowth quantified. During CIL migration ceases when one explant touches the other. When CIL is impaired, an increase in overlap is observed ${ }^{25,27,31,49}$. A variant is to quantify overlapping of individual cell protrusions under higher magnification, allowing for more detailed behaviour analysis ${ }^{27,48}$. An alternative is quantification of nuclei overlap ${ }^{1}$, however CIL reduction does not always lead to nuclei overlapping making this assay less sensitive to subtle effects.

Radial Outgrowth. Explants of cells undergoing CIL radially disperse as this is the most efficient way for cells to spread. Cell dispersion can then be quantified by measuring the distance between neighbouring cells, as this is higher in cells undergoing $\mathrm{CIL}^{27}$.

Kinematics in 2D. Cells are cultured on a 2D substrate (or examined in vivo if 2D descriptions are relevant). During CIL, cells alter their motion, which results in changes in velocity and acceleration. Note that velocity and acceleration are vectors and therefore measure changes in both speed and direction. Care must be taken to compare with freely moving cells to highlight CIL specific effects. It is also important to meaningfully pool data (e.g. normalize to movement at the time of collision) or else one will observe a random collection of vectors as collisions occur in many orientations. This is a powerful assay to describe many aspects of the motion changes surrounding collisions ${ }^{25,26,58,96}$.

Kinematics in 1D. Cells are cultured on stripes of extracellular matrix and analysed similar to 2D. This increases the chance of collisions while limiting the degrees of freedom of motion, which eases interpretation. However, care must be taken to choose an optimal width of the micropattern as migration can be greatly affected ${ }^{97}$. There are a number of possible outcomes in 1D: repolarization and migration of the two cells away from each other, a non-mutual response with only one cell repolarizing, cells remaining in contact or migrating past or over each other ${ }^{23,71,98}$. 


\section{Box 2 Mathematical models of CIL explain social cell behaviour}

Through studying CIL Abercrombie developed a number of approaches to quantify the 'social behaviour' of cells in tissue culture, leading eminent scientists to call him "the pioneer ethologist of cells" ${ }^{\prime 99}$. He led cell biologists away from qualitative analyses and towards rigorous quantitation ${ }^{100}$, and he would certainly be inspired by the numerous mathematical models recently developed to investigate CIL. These models have been essential in highlighting how this seemingly simple reaction can explain the emergent social behaviour of a population of cells, which lead to responses such as collective cell migration. It is unclear precisely why there has been a sudden increase in CIL models, but it is possible that cell biologists are taking a page from real ethologists who have developed mathematical models to explain the collective motion of animals. Three simple rules are required to explain animal flocking behavior ${ }^{101}$ : cohesion of the group, alignment of motion, and separation (i.e. short range repulsion akin to $\mathrm{CIL}$ behaviour), and interestingly many of the CIL collective motion models use similar parameters. These models have been used to illustrate 3 types of coordinated cellular motion:

Spontaneous collective migration. Cells in culture often exhibit emergent coordinated patterns of movement such as swirling and streaming ${ }^{102}$, which until recently have been largely unexplained. Modelling has revealed that when one takes into account the inherent cellular behaviours involved in CIL (e.g. cell repolarization ${ }^{21}$ ) within cells interacting within a population, coordinated movements can spontaneously emerge ${ }^{21,23,103-106}$. In these cases it is critical that CIL be integrated with other intercellular interactions, such as cellcell adhesion ${ }^{23,105}$ or chemotactic coattraction ${ }^{106}$. Furthermore, CIL can lead to other features observed during collective cellular motion, such as the patterns of traction stress $^{107}$.

Collective chemotaxis. There are many examples during development where large groups of cells need to collectively migrate towards some external cue, and it is unclear how such populations of cells organize and move in a coordinated fashion. Models have revealed the CIL behaviour within a population can greatly increase the efficiency of this coordinated chemotaxis. Again, CIL must be integrated with other factors such as coattraction $^{108,109}$, or confinement ${ }^{110}$ in order to collectively sense and migrate towards a chemotactic cue. In a recent twist, modelling revealed that an extracellular gradient capable of specifically modifying the strength of CIL between cells depending on the local 
concentration of the cue is enough to generate collective chemotaxis ${ }^{111}$. In this case the chemotactic cue is controlling directed migration by specifically modifying CIL properties rather than directly controlling the migratory machinery of the cells.

Cellular dispersion and tiling. One final type of coordinated motion that modelling has revealed to involve CIL is the dispersion of a population of cells. In this case, simple rules controlling cell collision and subsequent repulsion are capable of 'driving' the spreading of the population ${ }^{40}$, and depending on the precision of the CIL response (i.e. the consistency of the repulsion between cells during collisions), can even lead to an even cellular distribution ${ }^{26,58}$. This type of coordinated cellular motion explains the radial outgrowth of cells from an explant observed by Abercrombie and colleagues. Indeed, the same simple CIL rules used in a previous kinematic model to explain cellular tiling ${ }^{58}$ is enough to simulate the radial outgrowth of cells from an explant (supplementary Movie 5). 


\section{Glossary}

Contact inhibition of locomotion. A cellular reaction involving a cessation of forward motion upon migratory collision with another cell.

Contact inhibition of proliferation. The reduced proliferation of cells upon increase in cellular density, which may or may not involve direct cell contact.

Homotypic CIL. CIL behaviours between cells of the same type.

Heterotypic CIL. CIL behaviours between different cell types.

Adherens junction. A cadherin-mediated cell-cell junction that is normally thought to mediate the stable adhesion of epithelial cells.

Leading edge (used synonymously with lamellae here). The front of a migrating cell that contains an actin network that pushes out the plasma membrane and is involved in generating the forces behind cell migration.

Neural crest cells. A transient embryonic cell type specific to vertebrates, which undergoes a number of developmental migrations before differentiating into diverse cell types, such as melanocytes, cartilage, and glia.

Epithelial mesenchymal transition. A process in which epithelial cells lose epithelial characteristics, such as their polarity and cell-cell adhesions, and gain characteristics thought specific to mesenchymal cells, such as enhance motility and invasiveness.

Cajal-Retzius cells. A transient neuronal population during embryogenesis that undergoes specific migrations in the brain and controls the development of other neuronal cells.

Small GTPases. A family of proteins that includes Rho, Rac, and CDC42, which control the regulation of the cytoskeleton.

Planar cell polarity pathway. The polarization of cells within a sheet in a planar fashion, which involves a core set of components involving a transmembrane protein, such as frizzled, and downstream signaling mediators, such as dishevelled. 
Eph-ephrin. A transmembrane receptor (Eph) and its membrane bound ligand (ephrin), which can signal bidirectionally (i.e. both receptor and ligand can induce signaling) to control the repulsive interaction between different cell types such as neuronal cells.

Slit-robo. A transmembrane receptor (Slit) and its normally secreted ligand (robo) largely studied in the context of neuronal growth cone guidance.

Formin. A family of proteins involved in polymerization of actin, which has been shown to regulate specific actin structures, and the organization of contractile cytoskeletal elements in cells such as stress fibres.

Interference reflection microscopy. A microscopy technique for cells cultured in vitro that uses polarized light to highlight cell structures close to the substrate, and was first used to highlight points of cell-substrate adhesion (i.e. focal adhesions).

Focal adhesions. Specific adhesions that anchor cells to the substrate, which contain a complex of signaling proteins, such as FAK and paxillin, along with transmembrane proteins such as integrins.

Collective cell migration. A collection of cells that is capable of coordinating their motility such that they move as a coherent group.

Chemotaxis. The response of cells to an extracellular chemical signal, which induces their migration in a directed fashion.

Neuronal growth cones. A dynamic, actin-rich structure at the end of axons that controls the migration nerve cells.

Dendritic fields. The development of an array of neuronal processes called dendrites in which individual cells cover specific, non overlapping spatial territories.

Mesenchymal cells. Cells of embryonic origin that exist in connective tissues throughout the body, which develop into a broad range of cell types, such as cartilage and bone. 


\section{Figure Legends}

Figure 1: Types of CIL behaviours and their outcomes. $a, b \mid$ Homotypic CIL interactions, which involve collisions between cells of the same type (cells 1 and 2). Collisions involving Type I CIL involve active cellular retraction resulting in a predominant movement away from the colliding partner (a). In contrast, collisions involving Type II CIL will simply cease moving upon contact with another cell or be randomly deflected around the collision. c, d | Heterotypic CIL interactions, which involve collisions between different cell types can also result in Type I (c) or Type II (d) responses.

Figure 2: Stages of CIL and their regulatory mechanisms. (includes Table).

Example of a CIL response involving stereotypical Type 1 behaviour, which ends in active repolarization. (1) The initial step in the response upon collision is the generation of a cellcell contact involving a range of possible receptors and classical cell adhesion molecules. (2) Subsequently, there will be a variable amount of protrusion inhibition and an alteration of GTPase signaling, which may directly affect protrusions by altering actin polymerization at the leading edge, or indirectly by a buildup in lamellar tension. Furthermore, there may be additional reorganization of the cytoskeleton such as a modification of microtubule dynamics or stability. (3) The cells will then begin to contract and repolarize (with the order of these events possibly cell type dependent $(3 a, 3 b)$, which will require further actomyosin contraction and a possible reorganization of focal adhesions. Finally, (4) the cell will migrate away from the colliding partner. It should be clear that these hypothetical stages will not be completely distinct, but overlap in time to allow for an integrated and seamless response.

Figure 3: Cadherin switching regulates EMT and CIL. Epithelial to mesenchymal transition (EMT) involves a gain in migratory capacity along with a reduction in cell-cell adhesion and this is controlled by a change in the type of cadherin expressed at the cell surface. E-cadherin is known to generate stable intercellular adhesions between epithelial cells. Upon loss of E-cadherin and acquisition of $\mathrm{N}$-cadherin, neural crest cells undergo EMT while simultaneously gaining a capacity for $\mathrm{CIL}^{27}$. E-cadherin suppresses EMT and CIL by signaling to other adhesion components, such as p-120 catenin, which polarizes the small GTPase Rac1 toward cell-cell junctions. In contrast, N-cadherin expression leads to polarized Rac1 activity towards the leading edge of cells in an epithelial sheet, 
which allows them to generate asymmetric traction stresses for directed migration away from neighbouring cells.

Figure 4: Embryological functions of CIL. a | CIL has been shown to drive the dispersion of a Cajal-Retzius cells during development ${ }^{40}$, a neuronal population in the cerebral cortex. The cells are initially clustered and due to their Eph-ephrin regulated CIL interactions they spread throughout the tissue in a manner similar to radial outgrowth from an explant (supplementary movie 5). b | CIL controls the even spacing of Drosophila macrophages (hemocytes) during their developmental dispersal, which requires precisely orchestrated CIL interactions ${ }^{26,58}$. This CIL precision is regulated by the coupling of the flowing actin networks between colliding lamellae, which leads to the development of intercellular tension ${ }^{26}$. c| Homotypic CIL interactions control the collective migration of neural crest cells during their migration ${ }^{25,109}$. In this case, CIL behaviour is integrated with other intercellular responses, such as co-attraction mediated by a chemoattactant, $\mathrm{C} 3 \mathrm{a}^{109}$, and chemotaxis ${ }^{49}$ (not shown here) to allow for coherent motion of the population. $d$ I Heterotypic CIL interactions also control the coordinated movement of neural crest and placodal cell populations ${ }^{61}$. The neural crest are attracted to placodal cells via placodal expression of the chemoattractant, Sdf1. Due to placodal CIL responses to neural crest, the placodal cells are 'driven' collectively in a response termed chase and run behaviour.

Figure 5: Unexplored roles for CIL in vivo. a | Repulsion between neuronal cells, which has recently been termed 'contact repulsion', involves cellular interactions analogous to the CIL described in this review. CIL may be involved in spacing neuronal cells bodies or preventing overlap of their dendrites to allow dendritic tiling ${ }^{77-80}$. b | Zebrafish stripe formation involves the spacing of different pigment cell populations requiring intercellular interactions that appear identical to $\mathrm{CIL}^{81-84} . \mathrm{c} \mid$ The formation of boundaries in the hindbrain, which involves segmentation of cell populations, has been hypothesized to require CIL-type behaviours that prevent intermixing ${ }^{45,85-87}$. d | Cartilage condensation involves the coalescence of cells in the mesenchyme, which may involve turning off $\mathrm{CIL}$ interactions ${ }^{88}$. e | Inflammatory cell activation requires immune cells to gather to sites of infection or wounding ${ }^{92,94}$, and their ability to gather at these sites has been hypothesized to involve a reduction in their CIL capacity ${ }^{93} . \mathrm{f} \mid \mathrm{A}$ loss of CIL in cancerous cells may be involved in metastasis by aiding their invasiveness into neighbouring tissues (i.e. loss of heterotypic CIL) ${ }^{10,14,16,41,42,52,69}$. It is also possible that enhanced spreading may be controlled by maintenance of CIL interactions between 
cancer cells (i.e. homotypic CIL), which would provide a driving force for the spreading of the cancer population in a fashion similar to $\mathrm{CIL}$ in cell dispersion ${ }^{69}$. Therefore, both a loss of heterotypic CIL and maintenance of homotypic CIL could be playing a role in the invasive potential of a cancer.

\section{Supplementary Movies}

\section{Supplementary movie 1}

Movies from the Abercrombie laboratory showing CIL between chick heart fibroblasts, which is an example of a cell type exhibiting Type I CIL behaviour. The low magnification view highlights the collision and subsequent migration away from the colliding partner, while the high magnification view shows the sudden recoil of colliding lamellae, suggesting the buildup and release of tension. Movies were restored and digitized with the help of the Wellcome Library (http://blog.wellcomelibrary.org/2014/09/cells-on-film-making-movies-inbiology/) ${ }^{100}$.

\section{Supplementary movie 2}

Timelapse movie of Drosophila macrophages developmentally dispersing within the embryo. Hemocytes contain fluorescently labelled microtubules along with a nuclear marker, which allows for automated tracking of cells. A single macrophage was tracked in the centre of the field, which revealed sudden reversals in direction upon collision with neighbouring cells and this repulsion is suggestive of Type II CIL behaviour. Note the relatively even spreading of cells within the field of view, which is controlled by CIL dynamics $^{26,58,91 .}$

\section{Supplementary movie 3}

Movies from the Abercrombie laboratory showing CIL between epithelial cells in culture. The low magnification view highlights the collision of two epithelial sheets in culture, while the high magnification view shows epithelial cell collision between dispersed cells.

Epithelial cells do not show active repolarization during CIL, which is suggestive of Type II CIL behaviour. Movies were restored and digitized with the help of the Wellcome Library (http://blog.wellcomelibrary.org/2014/09/cells-on-film-making-movies-in-biology/) ${ }^{100}$. 


\section{Supplementary movie 4}

Movies from the Abercrombie laboratory showing a loss of CIL between $\mathrm{S} 180$ cells (sarcoma cells) and fibroblasts. Note that S-180 cells show a complete failure of CIL behaviour towards fibroblasts and are capable of using these cells as a substrate for their motility. In contrast, $\mathrm{S} 180$ cells still show CIL towards each other revealing a maintenance of homotypic CIL despite losing heterotypic CIL towards fibroblasts. Movies were restored and digitized with the help of the Wellcome Library (http://blog.wellcomelibrary.org/2014/09/cells-on-film-making-movies-in-biology/) ${ }^{100}$.

\section{Supplementary movie 5}

Simulation of outgrowth of an explant of cells in culture. Left panel shows cell positions, while right panel shows cell tracks. Cells were assumed to migrate with a biased random walk behaviour. However, when within a defined distance to a neighbouring cell, a CIL response occurs leading to repulsion. These simple rules lead to spreading and radial outgrowth of simulated cells from the explant, which is similar to the behaviour of real explants in vitro. The precise parameters for this simulation were taken from mathematical modelling of dispersing Drosophila macrophages ${ }^{58}$. 


\section{References}

1. Abercrombie, M. \& Heaysman, J.E. Observations on the social behaviour of cells in tissue culture. II. Monolayering of fibroblasts. Exp Cell Res 6, 293-306 (1954).

This is the first publication that coins the term contact inhibition.

2. Danuser, G., Allard, J. \& Mogilner, A. Mathematical modeling of eukaryotic cell migration: insights beyond experiments. Annu Rev Cell Dev Biol 29, 501-28 (2013).

3. Stramer, B.M., Dunn, G.A., Davis, J.R. \& Mayor, R. Rediscovering contact inhibition in the embryo. J Microsc 251, 206-11 (2013).

4. Taylor, D.L. \& Wang, Y.L. Molecular cytochemistry: incorporation of fluorescently labeled actin into living cells. Proc Natl Acad Sci U S A 75, 857-61 (1978).

5. Stoker, M.G. Role of diffusion boundary layer in contact inhibition of growth. Nature 246, 200-3 (1973).

6. Stoker, M. \& Piggott, D. Shaking 3T3 cells: further studies on diffusion boundary effects. Cell 3, 207-15 (1974).

7. Dunn, G.A. \& Ireland, G.W. New evidence that growth in 3T3 cell cultures is a diffusionlimited process. Nature 312, 63-5 (1984).

8. Stoker, M.G. \& Rubin, H. Density dependent inhibition of cell growth in culture. Nature 215, 171-2 (1967).

9. Takai, Y., Miyoshi, J., Ikeda, W. \& Ogita, H. Nectins and nectin-like molecules: roles in contact inhibition of cell movement and proliferation. Nat Rev Mol Cell Biol 9, 603-15 (2008).

10. Abercrombie, M. Contact inhibition and malignancy. Nature 281, 259-62 (1979).

11. Abercrombie, M. Contact inhibition in tissue culture. In vitro 6, 128-42 (1970).

12. Martz, E. \& Steinberg, M.S. Contact inhibition of what? An analytical review. J Cell Physiol 81, 25-37 (1973).

13. Steinberg, M.S. \& Garrod, D.R. Observations on the sorting-out of embryonic cells in monolayer culture. J Cell Sci 18, 385-403 (1975).

14. Abercrombie, M. Contact inhibition: the phenomenon and its biological implications. Natl Cancer Inst Monogr 26, 249-77 (1967).

15. Abercrombie, M. The Croonian Lecture, 1978: The crawling movement of metazoan cells. Proc. R. Soc. Lond. B 207:129-147 (1980).

16. Abercrombie, M. Control mechanisms in cancer. European journal of cancer 6, 7-13 (1970).

17. Vesely, P. \& Weiss, R.A. Cell locomotion and contact inhibition of normal and neoplastic rat cells. Int J Cancer 11, 64-76 (1973).

18. Mendoza, M.C., Vilela, M., Juarez, J.E., Blenis, J. \& Danuser, G. ERK reinforces actin polymerization to power persistent edge protrusion during motility. Sci Signal 8, ra47 (2015).

19. Ji, L., Lim, J. \& Danuser, G. Fluctuations of intracellular forces during cell protrusion. Nat Cell Biol 10, 1393-400 (2008).

20. Bohnet, S., Ananthakrishnan, R., Mogilner, A., Meister, J.J. \& Verkhovsky, A.B. Weak force stalls protrusion at the leading edge of the lamellipodium. Biophys J 90, 1810-20 (2006).

21. Camley, B.A. et al. Polarity mechanisms such as contact inhibition of locomotion regulate persistent rotational motion of mammalian cells on micropatterns. Proc Natl Acad Sci U S A 111, 14770-5 (2014).

22. Abercrombie, M. \& Dunn, G.A. Adhesions of fibroblasts to substratum during contact inhibition observed by interference reflection microscopy. Exp Cell Res 92, 57-62 (1975). 
23. Desai, R.A., Gopal, S.B., Chen, S. \& Chen, C.S. Contact inhibition of locomotion probabilities drive solitary versus collective cell migration. J R Soc Interface 10, 20130717 (2013).

Uses a combination of experiment and modeling to show that CIL is capable of controlling collective cellular motion.

24. Trinkaus, J.P., Betchaku, T. \& Krulikowski, L.S. Local inhibition of ruffling during contact inhibition of cell movement. Exp Cell Res 64, 291-300 (1971).

25. Carmona-Fontaine, C. et al. Contact inhibition of locomotion in vivo controls neural crest directional migration. Nature 456, 957-61 (2008).

This is the first example of cells undergoing CIL in vivo, and shows that RhoA and PCP signalling are involved.

26. Davis, J.R. et al. Inter-cellular forces orchestrate contact inhibition of locomotion. Cell 161, 361-73 (2015).

Shows that for CIL to work as a patterning cue requires precisely orchestrated repulsion, which for Drosophila macrophages involves intercellular forces.

27. Scarpa, E. et al. Cadherin Switch during EMT in Neural Crest Cells Leads to Contact Inhibition of Locomotion via Repolarization of Forces. Dev Cell 34, 421-34 (2015).

Reveals that switching from E- to N-Cadherin in neural crest cells during their EMT regulates their capacity for CIL.

28. Abercrombie, M. \& Ambrose, E.J. Interference microscope studies of cell contacts in tissue culture. Exp Cell Res 15, 332-45 (1958).

29. Heaysman, J.E. \& Pegrum, S.M. Early contacts between fibroblasts. An ultrastructural study. Exp Cell Res 78, 71-8 (1973).

30. Abbruzzese, G., Becker, S.F., Kashef, J. \& Alfandari, D. ADAM13 cleavage of cadherin-11 promotes CNC migration independently of the homophilic binding site. Dev Biol (2015).

31. Becker, S.F., Mayor, R. \& Kashef, J. Cadherin-11 mediates contact inhibition of locomotion during Xenopus neural crest cell migration. PLoS One 8, e85717 (2013).

32. Bracke, M.E. et al. Functional downregulation of the E-cadherin/catenin complex leads to loss of contact inhibition of motility and of mitochondrial activity, but not of growth in confluent epithelial cell cultures. Eur J Cell Biol 74, 342-9 (1997).

33. Chen, W.C. \& Obrink, B. Cell-cell contacts mediated by E-cadherin (uvomorulin) restrict invasive behavior of L-cells. J Cell Biol 114, 319-27 (1991).

34. Huttenlocher, A. et al. Integrin and cadherin synergy regulates contact inhibition of migration and motile activity. J Cell Biol 141, 515-26 (1998).

35. Omelchenko, T. et al. Contact interactions between epitheliocytes and fibroblasts: formation of heterotypic cadherin-containing adhesion sites is accompanied by local cytoskeletal reorganization. Proc Natl Acad Sci U S A 98, 8632-7 (2001).

36. Gloushankova, N.A. et al. Dynamics of contacts between lamellae of fibroblasts: essential role of the actin cytoskeleton. Proc Natl Acad Sci U S A 95, 4362-7 (1998).

37. Thiery, J.P., Acloque, H., Huang, R.Y. \& Nieto, M.A. Epithelial-mesenchymal transitions in development and disease. Cell 139, 871-90 (2009).

38. Seidel, B., Braeg, S., Adler, G., Wedlich, D. \& Menke, A. E- and N-cadherin differ with respect to their associated p120ctn isoforms and their ability to suppress invasive growth in pancreatic cancer cells. Oncogene 23, 5532-42 (2004).

39. Kania, A. \& Klein, R. Mechanisms of ephrin-Eph signalling in development, physiology and disease. Nat Rev Mol Cell Biol 17, 240-56 (2016). 
40. Villar-Cervino, V. et al. Contact repulsion controls the dispersion and final distribution of Cajal-Retzius cells. Neuron 77, 457-71 (2013).

Shows how CIL is controlling the dispersion of neuronal cells in vivo through Eph/ephrin signaling.

41. Astin, J.W. et al. Competition amongst Eph receptors regulates contact inhibition of locomotion and invasiveness in prostate cancer cells. Nat Cell Biol 12, 1194-204 (2010).

Shows how distinct Eph signaling controls the CIL capacity between cancer cells and normal cells to control their invasiveness.

42. Batson, J., Maccarthy-Morrogh, L., Archer, A., Tanton, H. \& Nobes, C.D. EphA receptors regulate prostate cancer cell dissemination through Vav2-RhoA mediated cell-cell repulsion. Biol Open 3, 453-62 (2014).

43. Batson, J., Astin, J.W. \& Nobes, C.D. Regulation of contact inhibition of locomotion by Eph-ephrin signalling. J Microsc 251, 232-41 (2013).

44. Solanas, G., Cortina, C., Sevillano, M. \& Batlle, E. Cleavage of E-cadherin by ADAM10 mediates epithelial cell sorting downstream of EphB signalling. Nat Cell Biol 13, 1100-7 (2011).

45. Fagotto, F., Rohani, N., Touret, A.S. \& Li, R. A molecular base for cell sorting at embryonic boundaries: contact inhibition of cadherin adhesion by ephrin/ Ephdependent contractility. Dev Cell 27, $72-87$ (2013).

46. Emerson, M.M. \& Van Vactor, D. Robo is Abl to block N-Cadherin function. Nat Cell Biol 4, E227-30 (2002).

47. Loveless, T. \& Hardin, J. Cadherin complexity: recent insights into cadherin superfamily function in C. elegans. Curr Opin Cell Biol 24, 695-701 (2012).

48. Moore, R. et al. Par3 controls neural crest migration by promoting microtubule catastrophe during contact inhibition of locomotion. Development 140, 4763-75 (2013).

49. Theveneau, E. et al. Collective chemotaxis requires contact-dependent cell polarity. Dev Cell 19, 39-53 (2010).

Shows how integrating CIL and chemotaxis responses can control the collective migration of a cell population.

50. Burridge, K. \& Wennerberg, K. Rho and Rac take center stage. Cell 116, 167-79 (2004).

51. Fritz, R.D. et al. SrGAP2-Dependent Integration of Membrane Geometry and Slit-RoboRepulsive Cues Regulates Fibroblast Contact Inhibition of Locomotion. Dev Cell 35, 7892 (2015).

52. Tanaka, M., Kuriyama, S. \& Aiba, N. Nm23-H1 regulates contact inhibition of locomotion which is affected by ephrin-B1. J Cell Sci (2012).

53. Kadir, S., Astin, J.W., Tahtamouni, L., Martin, P. \& Nobes, C.D. Microtubule remodelling is required for the front-rear polarity switch during contact inhibition of locomotion. $J$ Cell Sci 124, 2642-53 (2011).

54. Abercrombie, M. \& Heaysman, J.E. Observations on the social behaviour of cells in tissue culture. I. Speed of movement of chick heart fibroblasts in relation to their mutual contacts. Exp Cell Res 5, 111-31 (1953).

55. Weber, G.F., Bjerke, M.A. \& DeSimone, D.W. A mechanoresponsive cadherin-keratin complex directs polarized protrusive behavior and collective cell migration. Dev Cell 22, 104-15 (2012).

56. Winklbauer, R., Selchow, A., Nagel, M. \& Angres, B. Cell interaction and its role in mesoderm cell migration during Xenopus gastrulation. Dev Dyn 195, 290-302 (1992). 
57. Nakatsuji, N. \& Johnson, K.E. Cell locomotion in vitro by Xenopus laevis gastrula mesodermal cells. Cell Motil 2, 149-61 (1982).

58. Davis, J.R. et al. Emergence of embryonic pattern through contact inhibition of locomotion. Development 139, 4555-60 (2012).

Uses a combination of experimentation and modeling to show that CIL dynamics alone can drive the patterned movement of cells in vivo.

59. Nagasaki, T., Chapin, C.J. \& Gundersen, G.G. Distribution of detyrosinated microtubules in motile NRK fibroblasts is rapidly altered upon cell-cell contact: implications for contact inhibition of locomotion. Cell Motil Cytoskeleton 23, 45-60 (1992).

60. Harris, A. Location of cellular adhesions to solid substrata. Dev Biol 35, 97-114 (1973).

61. Theveneau, E. et al. Chase-and-run between adjacent cell populations promotes directional collective migration. Nat Cell Biol 15, 763-72 (2013).

Shows how heterotypic CIL interactions can control the morphogenesis of tissues.

62. McCain, M.L., Lee, H., Aratyn-Schaus, Y., Kleber, A.G. \& Parker, K.K. Cooperative coupling of cell-matrix and cell-cell adhesions in cardiac muscle. Proc Natl Acad Sci U S A 109, 9881-6 (2012).

63. de Rooij, J., Kerstens, A., Danuser, G., Schwartz, M.A. \& Waterman-Storer, C.M. Integrindependent actomyosin contraction regulates epithelial cell scattering. J Cell Biol 171, 153-64 (2005).

64. Yamada, S. \& Nelson, W.J. Localized zones of Rho and Rac activities drive initiation and expansion of epithelial cell-cell adhesion. J Cell Biol 178, 517-27 (2007).

65. Tseng, Q. et al. Spatial organization of the extracellular matrix regulates cell-cell junction positioning. Proc Natl Acad Sci U S A 109, 1506-11 (2012).

66. Maruthamuthu, V., Sabass, B., Schwarz, U.S. \& Gardel, M.L. Cell-ECM traction force modulates endogenous tension at cell-cell contacts. Proc Natl Acad Sci U S A 108, 470813 (2011).

67. Martinez-Rico, C., Pincet, F., Thiery, J.P. \& Dufour, S. Integrins stimulate E-cadherinmediated intercellular adhesion by regulating Src-kinase activation and actomyosin contractility. J Cell Sci 123, 712-22 (2010).

68. Weber, G.F., Bjerke, M.A. \& DeSimone, D.W. Integrins and cadherins join forces to form adhesive networks. J Cell Sci 124, 1183-93 (2011).

69. Abercrombie, M. \& Ambrose, E.J. The surface properties of cancer cells: a review. Cancer Res 22, 525-48 (1962).

70. Fairen, A., Morante-Oria, J. \& Frassoni, C. The surface of the developing cerebral cortex: still special cells one century later. Prog Brain Res 136, 281-91 (2002).

71. Lin, B., Yin, T., Wu, Y.I., Inoue, T. \& Levchenko, A. Interplay between chemotaxis and contact inhibition of locomotion determines exploratory cell migration. Nat Commun 6, 6619 (2015).

72. Song, H. \& Poo, M. The cell biology of neuronal navigation. Nat Cell Biol 3, E81-8 (2001).

73. Tessier-Lavigne, M. \& Goodman, C.S. The molecular biology of axon guidance. Science 274, 1123-33 (1996).

74. Dunn, G.A. Mutual contact inhibition of extension of chick sensory nerve fibres in vitro. J Comp Neurol 143, 491-507 (1971).

75. Ebendal, T. The relative roles of contact inhibition and contact guidance in orientation of axons extending on aligned collagen fibrils in vitro. Exp Cell Res 98, 159-69 (1976).

76. Dunn, G.A. Extension of nerve fibres, their mutual interaction and direction of growth in tissue culture. Ciba Found Symp 14, 211-32 (1973). 
77. Cook, J.E. \& Chalupa, L.M. Retinal mosaics: new insights into an old concept. Trends Neurosci 23, 26-34 (2000).

78. Kay, J.N., Chu, M.W. \& Sanes, J.R. MEGF10 and MEGF11 mediate homotypic interactions required for mosaic spacing of retinal neurons. Nature 483, 465-9 (2012).

79. Grueber, W.B. \& Sagasti, A. Self-avoidance and tiling: Mechanisms of dendrite and axon spacing. Cold Spring Harb Perspect Biol 2, a001750 (2010).

80. Matthews, B.J. et al. Dendrite self-avoidance is controlled by Dscam. Cell 129, 593-604 (2007).

81. Yamanaka, H. \& Kondo, S. In vitro analysis suggests that difference in cell movement during direct interaction can generate various pigment patterns in vivo. Proc Natl Acad Sci U S A 111, 1867-72 (2014).

82. Inaba, M., Yamanaka, H. \& Kondo, S. Pigment pattern formation by contact-dependent depolarization. Science 335, 677 (2012).

83. Nakamasu, A., Takahashi, G., Kanbe, A. \& Kondo, S. Interactions between zebrafish pigment cells responsible for the generation of Turing patterns. Proc Natl Acad Sci US A 106, 8429-34 (2009).

84. Walderich, B., Singh, A.P., Mahalwar, P. \& Nusslein-Volhard, C. Homotypic cell competition regulates proliferation and tiling of zebrafish pigment cells during colour pattern formation. Nat Commun 7, 11462 (2016).

85. Cayuso, J., Xu, Q. \& Wilkinson, D.G. Mechanisms of boundary formation by Eph receptor and ephrin signaling. Dev Biol 401, 122-31 (2015).

86. Fagotto, F., Winklbauer, R. \& Rohani, N. Ephrin-Eph signaling in embryonic tissue separation. Cell Adh Migr 8, 308-26 (2014).

87. Rohani, N., Parmeggiani, A., Winklbauer, R. \& Fagotto, F. Variable combinations of specific ephrin ligand/Eph receptor pairs control embryonic tissue separation. PLoS Biol 12, e1001955 (2014).

88. Hall, B.K. \& Miyake, T. All for one and one for all: condensations and the initiation of skeletal development. Bioessays 22, 138-47 (2000).

89. Oldfield, F.E. Orientation behavior of chick leucocytes in tissue culture and their interactions with fibroblasts. Exp Cell Res 30, 125-38 (1963).

90. Armstrong, P.B. \& Lackie, J.M. Studies of intercellular invasion in vitro using rabbit peritoneal neutrophil granulocytes (PMNS). I. Role of contact inhibition of locomotion. J Cell Biol 65, 439-62 (1975).

91. Stramer, B. et al. Clasp-mediated microtubule bundling regulates persistent motility and contact repulsion in Drosophila macrophages in vivo. J Cell Biol 189, 681-9 (2010).

92. Stramer, B. et al. Live imaging of wound inflammation in Drosophila embryos reveals key roles for small GTPases during in vivo cell migration.J Cell Biol 168, 567-73 (2005).

93. Weavers, H. et al. Systems analysis of the dynamic inflammatory response to tissue damage reveals spatio-temporal properties of the wound attractant gradient. Curr Biol In Press (2016).

94. Lammermann, T. et al. Neutrophil swarms require LTB4 and integrins at sites of cell death in vivo. Nature 498, 371-5 (2013).

95. Ellenbroek, S.I. \& van Rheenen, J. Imaging hallmarks of cancer in living mice. Nat Rev Cancer 14, 406-18 (2014).

96. Dunn, G.A. \& Paddock, S.W. Analysing the motile behaviour of cells: a general approach with special reference to pairs of cells in collision. Philos Trans R Soc Lond B Biol Sci 299, 147-57 (1982).

97. Doyle, A.D., Wang, F.W., Matsumoto, K. \& Yamada, K.M. One-dimensional topography underlies three-dimensional fibrillar cell migration. J Cell Biol 184, 481-90 (2009). 
98. Scarpa, E. et al. A novel method to study contact inhibition of locomotion using micropatterned substrates. Biol Open 2, 901-6 (2013).

99. Dunn, G. \& Jones, G. Michael Abercrombie: the pioneer ethologist of cells. Trends Cell Biol 8, 124-6 (1998).

100. Stramer, B.M. \& Dunn, G.A. Cells on film - the past and future of cinemicroscopy.J Cell Sci 128, 9-13 (2015).

101. Reynolds, C. Flocks, herds and schools: A distributed behavioral model. Proceeding SIGGRAPH '87 Proceedings of the 14th annual conference on computer graphics and interactive techniques, 25-34 (1987).

102. Rorth, P. Fellow travellers: emergent properties of collective cell migration. EMBO Rep 13, 984-91 (2012).

103. Coburn, L., Cerone, L., Torney, C., Couzin, I.D. \& Neufeld, Z. Tactile interactions lead to coherent motion and enhanced chemotaxis of migrating cells. Phys Biol 10, 046002 (2013).

104. Landman, K.A., Fernando, A.E., Zhang, D. \& Newgreen, D.F. Building stable chains with motile agents: Insights into the morphology of enteric neural crest cell migration. $J$ Theor Biol 276, 250-68 (2011).

105. Lober, J., Ziebert, F. \& Aranson, I.S. Collisions of deformable cells lead to collective migration. Sci Rep 5, 9172 (2015).

106. Vedel, S., Tay, S., Johnston, D.M., Bruus, H. \& Quake, S.R. Migration of cells in a social context. Proc Natl Acad Sci U S A 110, 129-34 (2013).

107. Zimmermann, J., Camley, B.A., Rappel, W.J. \& Levine, H. Contact inhibition of locomotion determines cell-cell and cell-substrate forces in tissues. Proc Natl Acad Sci U S A 113, 2660-5 (2016).

108. Woods, M.L. et al. Directional collective cell migration emerges as a property of cell interactions. PLoS One 9, e104969 (2014).

109. Carmona-Fontaine, C. et al. Complement Fragment C3a Controls Mutual Cell Attraction during Collective Cell Migration. Dev Cell (2011).

110. Szabo, A. et al. In vivo confinement promotes collective migration of neural crest cells. $J$ Cell Biol 213, 543-55 (2016).

111. Camley, B.A., Zimmermann, J., Levine, H. \& Rappel, W.J. Emergent Collective Chemotaxis without Single-Cell Gradient Sensing. Phys Rev Lett 116, 098101 (2016). 
CIL

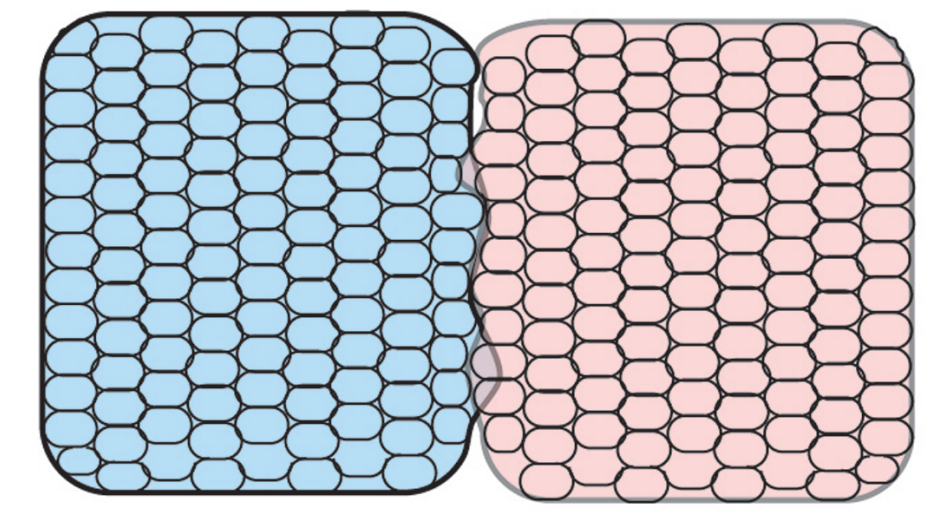

Inhibition of CIL

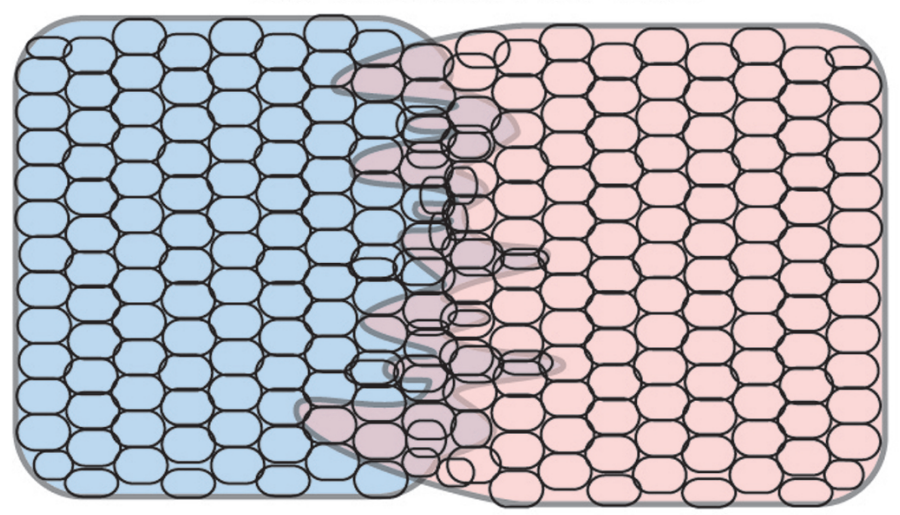

B CIL

Radial Outgrowth

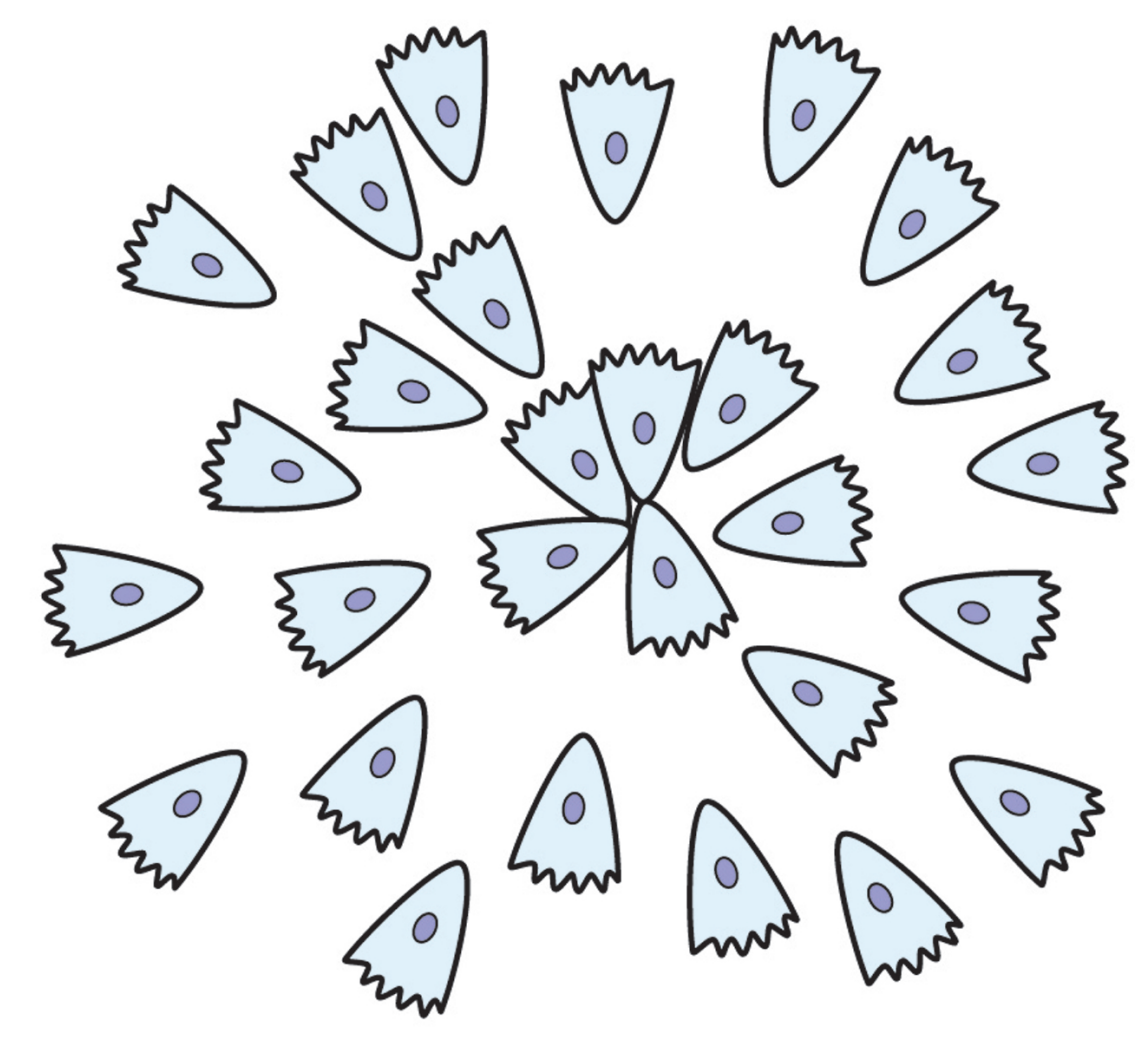

Inhibition ofCIL

C

Kinematics in 2D
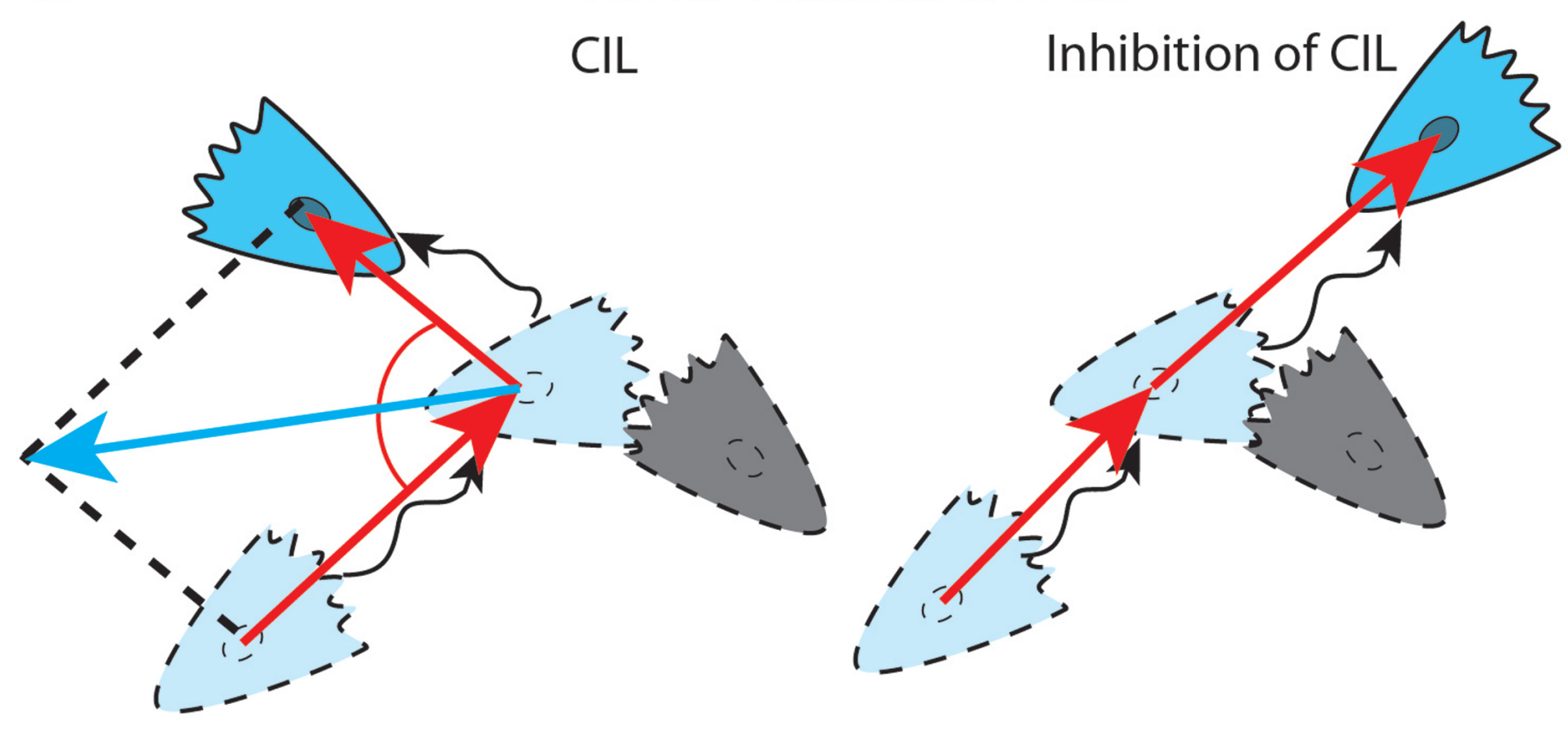

D

Kinematics in 1D

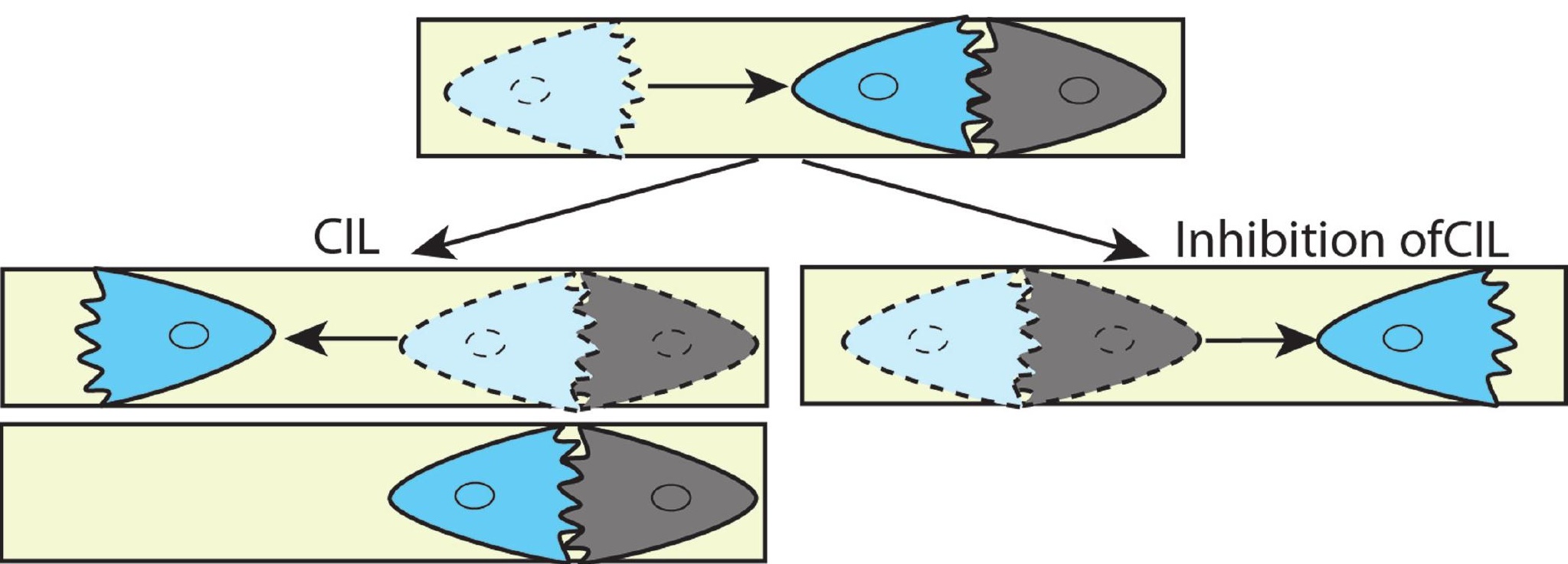


Homotypic

Heterotypic

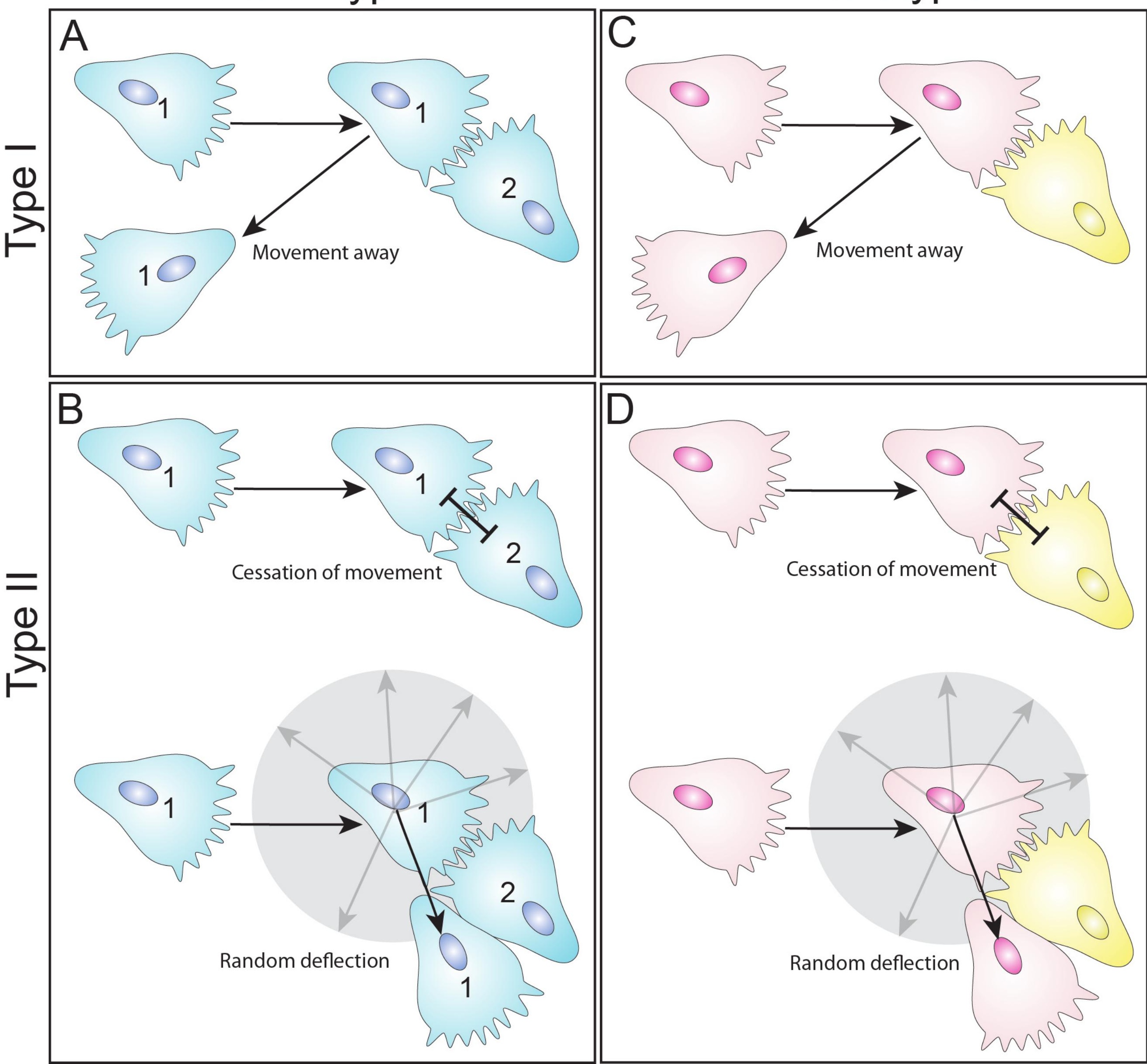




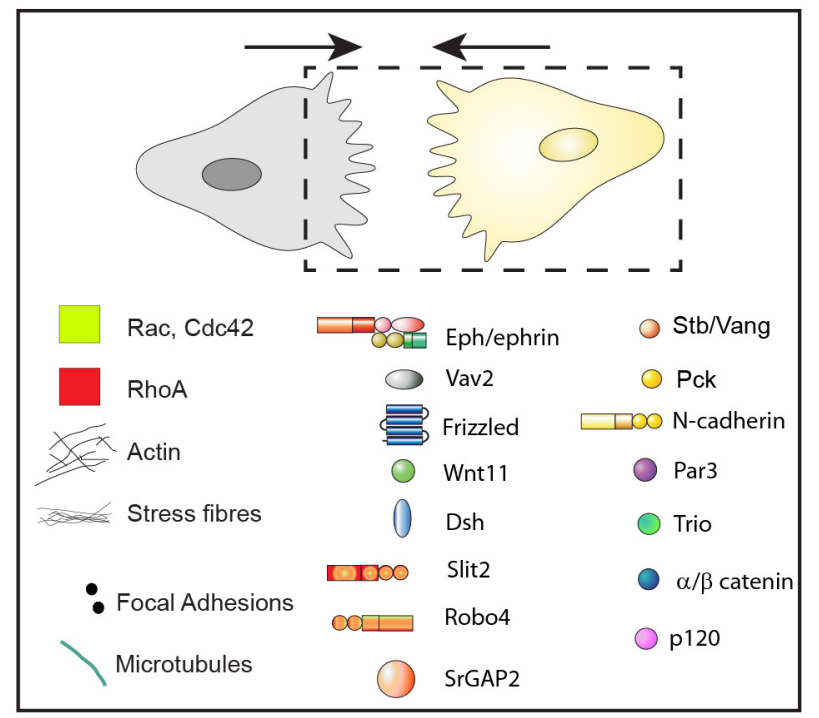

1. Cell-cell contact

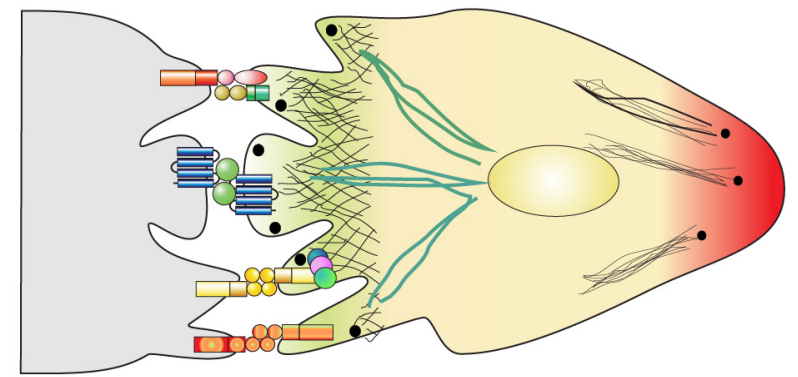

2. Protrusion inhibition

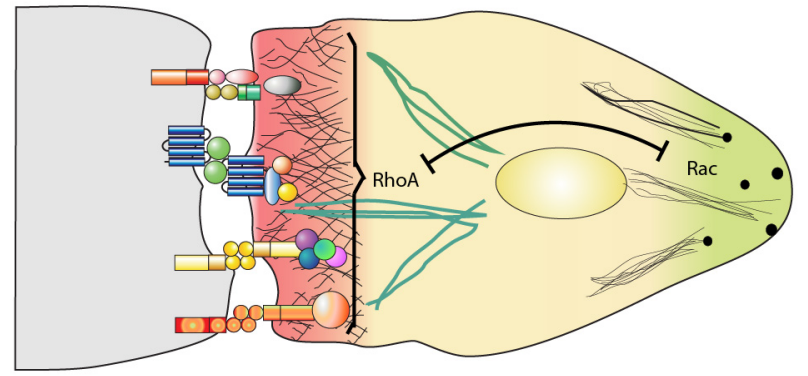

3a. Polarization and contraction
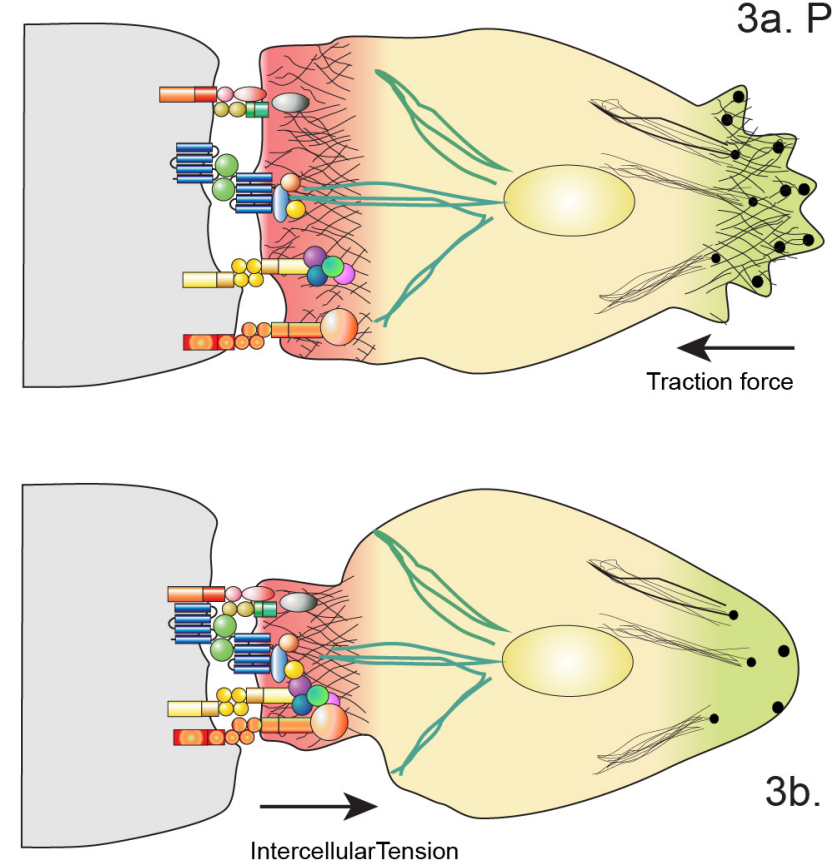

3b. Contraction and repolarization
4. Migration away
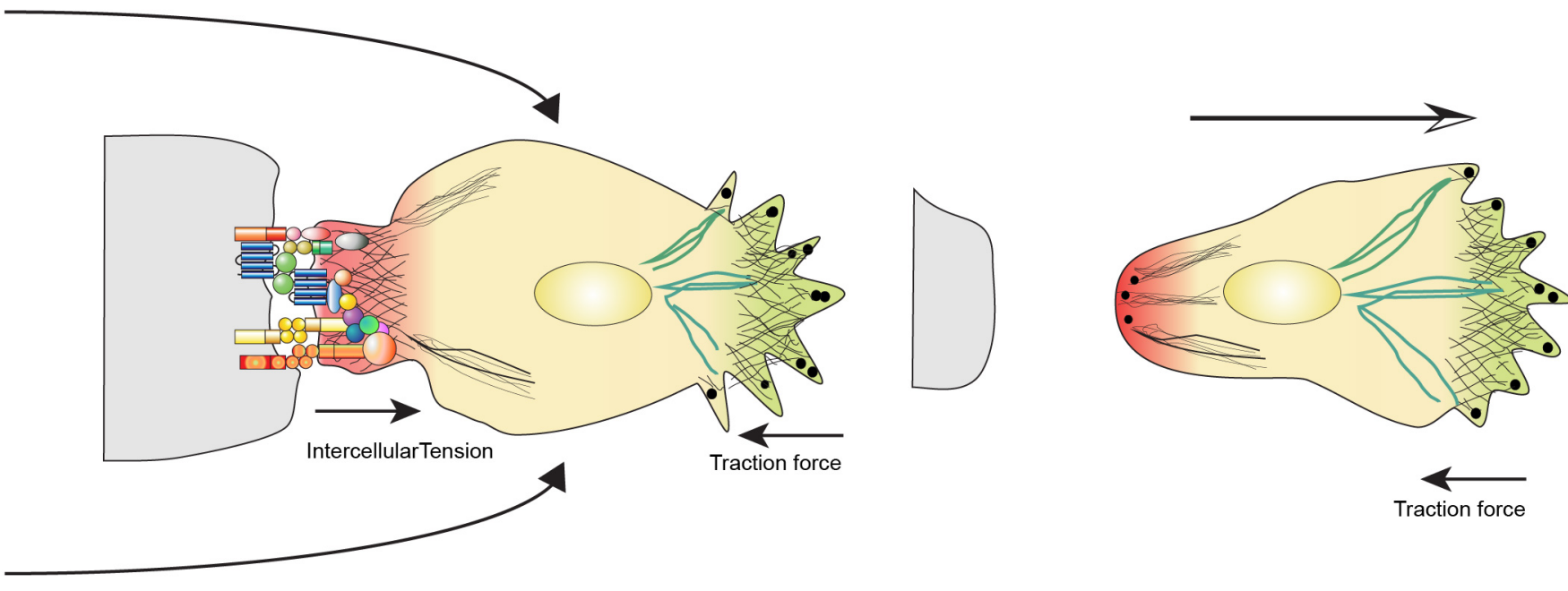

Indications for Artist

otolit! It

Replace Eph/ephrins with a more realistic reprsentation (previously published in NRMCB Use one color for Ephrin (blue in the example) and another color with shades for Eph

Replace Cadherin with a more realistic representation (previoulsy published in NRMCB

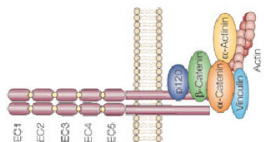

Replace Slit/Robo with a more realistic representation

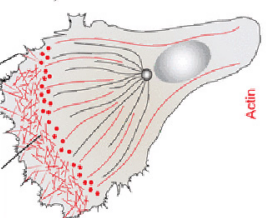


EPITHELIAL

Cadherin switch

\section{EMT}

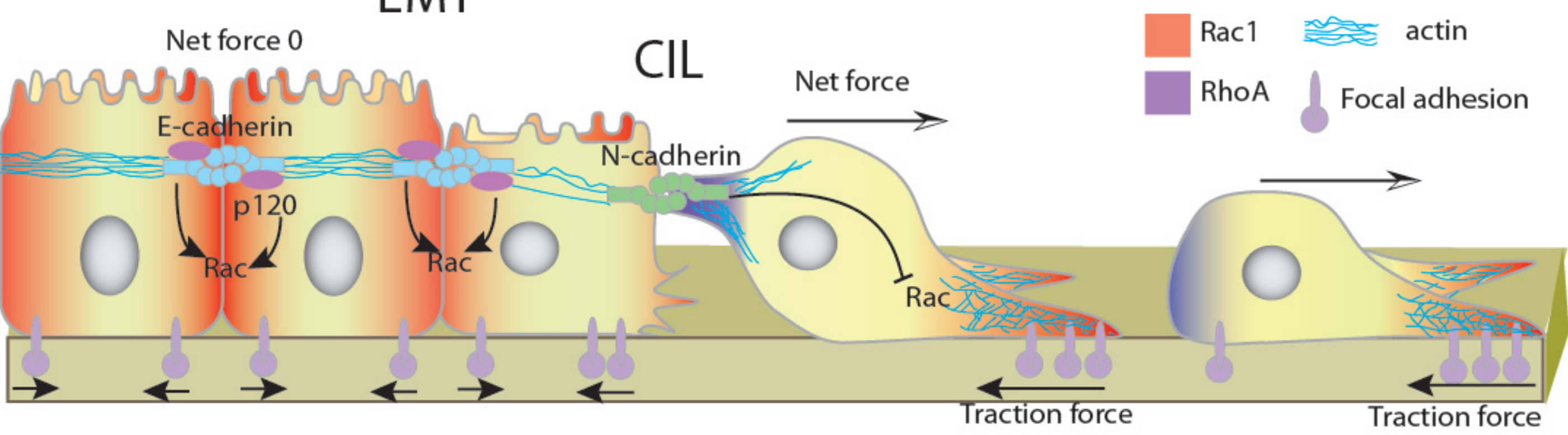

Notes for artist:

Improve representation of $\mathrm{E}-$ and $\mathrm{N}$ - cadherin. For example:

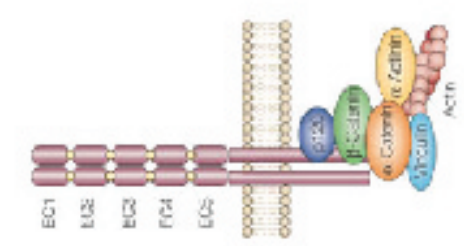

Improve representtion of extrecalular matrix and focal adhesions (or intergrins) 
